sepsis facilitates the diagnosis. Myocarditis is likely to be overlooked in cases where the primary disorders which produce the complaint have passed unnoticed, as is very likely to happen and as a matter of fact frequently does happen, and when the patient first comes under observation as a case of heart disease, especially if the condition some. times happens to be associated with cardiac bruits independent of valvular lesions. Of cases of temporary cardiac invalidism there must be many examples which escape detection. Now that attention is drawn to the condition it is hoped that it may bring forth valuable observations in the future on an important subject which needs further and more extended investigations.

Welbeck-street, $\mathrm{W}$.

\section{A CASE OF ANILINE POISONING.}

\section{BY P. G. HARVEY, M.B. LoND., L.R.C.P. LoNd, M.R.C.S. ENG.,}

LATE HOUSE PHYSICIAN A'I' IHE WORCESTER GENERAL INFIRMARY.

A MARRIED woman, aged 27 years, was admitted into the General Infirmary, Worcester, on May 10th, 1904, under the care of Dr. Mabyn Read, with the following history. For the last two months she had suffered from a painful swelling of the lips and gums, the pain being so severe as to prevent her taking solid food. 'l'here was no increased salivation. For the last two months she had also suffered from considerable shortness of breath and a feeling of faintness. A fortnight after the onset of these symptoms she noticed a discolouration of the gums and the inner side of the cheeks. About this time she began to suffer from sickness; vomiting occurred immediately after taking food which was returned unaltered. The nature of the food taken made no difference to the sickness and there was no abdominal pain, diarrhœe, or constipation. The patient said that her urine was very dark in colour about this time. She did not remember taking any food which upset her, nor had anyone else in her house suffered from similar symptoms. Six weeks previously she came to the infirmary as an outpatient but for family reasons would not become an inpatient; she only attended twice and since then had been obliged to keep indoors and to lie down on account of the shortness of breath. She had been attended by her private medical adviser for the last six weeks. During the last week her general condition had improved and she had not had any sickness. The past history and family history were unimportant.

On admission the patient was pale but not markedly anæmic-looking. Her complexion was somewhat sallow. The breath was offensive and the tongue was coated with a brownish fur. On examining the gums the first thing to attract attention was the pigmentation which was situated at the alveolar margin ; it was dark blue in colour, being not unlike the pigmentation seen in lead poisoning but rather brighter in colour. Unlike the "line" of lead poisoning it was more like a band of pigmentation, as it extended further over the gums, in some places being fully a third of an inch deep, gradually fading into the normal colour of the gum. It was most marked on the labial aspect of the gum but was also present on the lingual aspect. It was especially noticeable around the upper left second molar which was very carious; it was also noticeable along the gum where several teeth had fallen out or had been removed some time before. On the mucous membrane of the cheeks, parallel with, and opposite to, the teeth of both the upper and lower jaw on both sides of the mouth were two bands of similar pigmentation, but the outline was much more wavy and about half an inch deep. These bands extended right round the mouth and behind on to the mucous membrane, covering the ascending ramus of the lower jaw. There was no pigmentation on the palate. All the organs were healthy. 'There was no sign of paralysis in the limbs. No increased pigmentation was found elsewhere on the body. The urine was of specific gravity 1018, acid, and contained no albumin.

A diagnosis of possible ptomaine poisoning or a local infection in the mouth from some unknown micro-organism was made. Lead poisoning was excluded by the absence of paralysis at the wrists, the presence of piomentation on the cheeks, and by the nature of the pigmentation on the gums. 'The sickness did not secm like the ordinary lead colic. Addison's discase suggested itsolf on account of the gastric symptoms from which the patient suffered, but the pigmentation was unlike that found in this disease and did not exist elsewhere. The treatment was purely symptomatic, a bismuth mixture and an antiseptic mouth wash which were used when she was an out-patient were continued, fluid food only being given. A culture from the mouth was not made at the time. One made later showed staphylococci and streptococci only.

On May 16th Dr. G. W. Crowe saw the patient and at once suggested aniline poisoning as a possible cause for her symptoms. On following up this idea it was found that she was in the habit of using an indelible pencil for writing, which she moistened in her mouth from time to time. She had used this class of pencil for years and did not think that she had used it more than usual lately. She promised to obtain the pencil but failed to do so. A similar pencil tested afterwards gave most of the tests for aniline. The urine was examined with negative results. The patient was discharged on May $28 \mathrm{th}$, the general symptoms having subsided and the pigmentation having steadily decreased, especially on the cheeks.

This case seems remarkable on account of the length of time during which the habit of sucking the pencil had existed without previously causing any symptoms. Dixon Mann ${ }^{1}$ mentions cases of aniline poisoning which resemble the above case fairly closely. When a large quantity was swallowed at one time vomiting, dizziness going on to coma, dyspncea, and intense blueness of the surface were prominent symptoms. Milder cases suffered from discolouration of the lips and face without dyspnoea and vomiting. $\mathrm{He}$ also quotes Rayner" who relates a series of cases which seem very similar. The aniline in these cases was absorbed from napkins which were marked with a stamp the ink of which was aniline. Locally the buttocks and vulva were stained. The children, who were newly born, had blue discolouration of the gums, lips, and palate; the skin was dusky but no general symptoms were noted.

I am greatly indebted to Dr. Read for permission to publish, and also for kindly revising an account of, this case. Penzance.

\section{CERVICAL FIBROID OF THE UTERUS OBSTRUCTING LABOUR; CASAREAN SECTION : SUPRAVAGINAL HYSTERECTOMY.}

By H. I. D. ACLAND, F.R.C.S. ENG.

HONORART SLRGFON, CHRISTCHURCH HOSPITAL, NEW TEALAND.

THE following case from its comparative rarity seems to me worthy of publication. The patient, aged 39 years, who had been married seven years, had reached full term of her third pregnancy. The first confinement occurred five years ago: the labour lasted seven hours and forceps were used. The second confinement took place two years and eight months previously; the labour lasted 12 hours and the child was born before the arrival of the medical attendant. After the second confinement until the third pregnancy commenced menstruation was regular and there was no increase in the amount lost at each period. In the early months of the third pregnancy there were no abnormal symptoms, but during the last six weeks she suffered discomfort and pain of a bearing-down character, so that she often thought labour was commencing. There was increased frequency of micturition and diarrhoea was present almost throughout the whole pregnancy.

Labour commenced on May 11th, 1904, at 2 A.M.; the pains were strong and regular throughout the day. Between 7 and 8 P.x. she was seen by Dr. J. Cooke of Lincoln who found on examination a large fibroid blocking the pelvis and immediately sent her to the Christchurch Hospital for operation. She was driven a distance of 12 miles in a dog. cart, kneeling on the floor of the vehicle with her arms resting on the seat, and arrived at the hospital at about 11 P.M.

On examination the abdomen presented the usual appearance seen at the end of pregnancy ; the uterine pains were so 1 Forensic Medicine and Toxicology, edition 1898, p. 561 . 
strong and followed one another so rapidly that but little could be made out by external palpation. Per vaginam a solid rounded mass was felt pressing down the posterior vaginal wall and occupying the whole of the posterior threefourths of the pelvis. High up behind the pelvis the os, dilated to admit four fingers, was felt. 'Ihe anterior lip was soft and cdematous; the posterior lip was not well defined but stretched out over the tumour. The membranes were unruptured and a foot could be felt presenting. Per rectum the posterior suface of the tumour was felt closely fitting the hollow of the sacrum. It was quite immoveable.

At $12.30 \mathrm{~A} . \mathrm{M}$. on May 12th, labour having been in progress for $20 \frac{1}{2}$ hours, Cæsarean section was performed. After delivery of the child the uterus was tilted through the abdominal incision and the placenta and membranes were removed. The bleeding was quite inconsiderable. The tumour was now ascertained to be a large fibroid springing from the posterior wall of the cervical canal and burrowing down into Douglas's pouch. It was decided to remove the uterus and fibroid. This was accomplished by ligaturing the ovarian arteries, dividing the broad ligaments, and raising peritoneal flaps from the anterior surface of the uterus and from the posterior surface of the fibroid behind. The tumour was easily shelled out from Douglas's pouch and with the uterus was brought out of the abdominal wound. The uterine arteries were then ligatured and the cervical canal was cut across near its lower end. The cervix was sutured with a few interrupted silk stitches, dropped back, and the peritoneum sutured over in the usual manner. The abdominal wound was closed in layers. The patient at the end of the operation, which lasted one hour and five minutes, was in good condition. The child weighed nine pounds and throve well. The mother made an uneventful recovery and left the hospital at the end of three and a half weeks.

The noticeable features in the operation were those which Amand Routh called attention to in his paper read before the British Medical Association in July, 1903-namely, the ease with which the peritoneum stripped up and with which the uterus and fibroid were drawn out of the abdomen; also the readiness with which the arteries, on account of their large size, were recognised and ligatured. There was some difficulty in deciding where exactly to divide the cervix, as owing to its canalisation it was not very clear where the cervix joined the vagina.

The parts removed consist of the uterus, both tubes and ovaries, with a fibroid springing from the posterior wall of the cervical canal and lower part of the body of the uterus. In the anterior wall of the uterus was an incision through which the child was extracted and below there was the dilated cervical canal cut across. The fibroid was ovoid in shape, the larger pole being above ; it sprang from the lower part of the posterior wall of the body of the aterus and from the posterior wall of the cervical canal and projected downwards below the level of the external os for about two inches. It measured eight inches in length and five inches in breadth. The circumference at the centre was $11 \frac{1}{2}$ inches, two inches above this it was $12 \frac{1}{2}$ inches, and two inches below the centre it was nine and a half inches. The whole length of the uterus and fibroid was 14 inches.

Christchurch, New Zealand.

\section{LITHOTRITY IN EGYPT FOR LARGE STONES.}

BY FRANK MILTON, M.R.C.S. ENG., L.R.C.P. LOND., PROFESSOR OF CLINICAL SURGERY, GOVERNMENT SCHOOL OF MEDICINE, CAIRO.

THE choice of operation in cases of small stones is not of great moment, for any form of operation should be successful, but when large stones have to be dealt with the operation decided upon will certainly affect the result. The fact that lithotrity, by which throughout this paper urethral lithotrity is meant, offers distinct advantages over other forms of treatment in all ordinary cases by reason of the rapidity of cure and the absence of after-suffering is firmly established, but it is not equally clear to all surgeons that lithotrity is the best form of operation in cases of stones of large sizethat is, of 50 grammes weight and over. Among the English surgeons in Cairo the doctrine is held that in all cases of stone in which the urethra is capable of taking a lithotrite of the pattern used by them lithotrity should be done through the urethra. The statistics which I am able to offer are taken from the practice of Mr. H. M. N. Milton and myself ; they concern 159 cases of stones of 50 grammes and over, of which $\mathrm{Mr}$. Milton contributes 113 and I 46, and they are consecutive cases and altogether unselected. All the patients were over 15 years of age with the exception of nine, of whom one 13 years of age was cured by a lithotrity, six between 12 and 14 years of age were cured by perineal lithotrity, and two between ten and 14 years of age were cured by suprapubic lithotomy. The cases treated by cutting operations were so treated either because that method was thought to be best for those particular cases at the time or because there was some mechanical difficulty other than the mere size of the stone preventing the grasping of the calculus with a lithotrite. The largest of all Mr. Milton's stones, which weighed 995 grammes, and for which laparotomy was successfully done, is not included. The largest stones treated under each heading were : by lithotrity, 452 grammes; by suprapubic lithotomy, 392 grammes; by perineal lithotrity, 387 grammes ; and by left lateral lithotomy, 90 grammes. The total results obtained were as follows:-Lithotrity: cases, 84 ; deaths, five; mortality, 5.9 per cent. Perineal lithotrity: cases, 47; deaths, seven; mortality, 14.9 per cent. Suprapubic lithotomy: cases, 22 ; deaths, eight ; mortality, $36 \cdot 3$ per cent. Left lateral lithotomy : cases, six ; deaths, nil ; mortality, nil. The comparative mortality of the two forms of operation, even including the lateral lithotomies which were all done for comparatively small stones, the largest only weighing 90 grammes, was, for lithotrity, $5 \cdot 9$ per cent. and for the cutting operations $18 \cdot 9$ per cent., showing a mortality in favour of crushing without a wound of 13 per cent.

Since the subject of a large stone must, from the nature of the disease, be in a certain percentage of cases the subject also of destructive disease of the kidneys, it follows that in all forms of operations undertaken for these cases there must be a certain proportion of, as it were, accidental mortalitythat is to say, cases which will die after any form of treatment. This being so and the gravity of the disease itself being taken into consideration the mortality of 5.9 per cent. will compare favourably with that normal to any operation performed for disease of like gravity. Lithotrity is essentially an operation which needs practice for its efficient performance and the results of which are directly in proportion to the experience of the operator, and the dexterity necessary for the attack. of large stones can only be obtained by constant practice on stones of ordinary size and weight. "Perineal lithotrity," as spoken of in this paper, is the operation described by Reginald Harrison, where the prostate is incised and the stone is broken up sufficiently to allow of its removal by forceps, and not the operation described by Keith where a small incision is made into the membranous urethra and the stone is crushed by a larger lithotrite than could be passed down the unwounded urethra and then washed out in the ordinary way. This last operation is hardly ever performed here, but the operation combining a perineal lithotomy with crushing of the stone with a special instrument is fairly common and after lithotrity gives the best results for large stones, but it has the drawback common to all cutting operations that in cases where the bladders are much disorganised by bilharzia the bladder symptoms are increased and owing to the bad nutrition of the part the wound is very long in healing and in some cases never heals at all, the patient dying even after a long interval with his wound in much the same condition as just after its infliction. There is an idea abroad that stones in Egypt are frequently very soft and that they occur in conjunction with chyluria; both these ideas are wrong, for although cases of soft phosphatic stones do occur in Egypt, as elsewhere, they are by no means abnormally frequent and the majority of stones will compare favourably, from the point of view of hardness, with those found in other countries; and also chyluria is a rare disease in this country and personally $I$ have never met with a case complicated with stone.

The instruments used at Kasr-el-Aini are of a pattern devised by Mr. Milton. The lithotrites differ from the ordinary pattern in the large diameter of the rotatory part of the handles and in the annular form of the slides, tending to give increased power and delicacy in crushing and to diminish fatigue. The evacuating syringe is of the 